\title{
Fatty Acid and Liver Status in Postmenopausal Women With and Without Metabolic Syndrome
}

\author{
Agata Muzsik $^{1}$, Henryk H. Jeleń ${ }^{2}$ and Agata Chmurzynska ${ }^{1}$ \\ ${ }^{1}$ Institute of Human Nutrition and Dietetics, Poznan University of Life Sciences, Poznań, Poland and \\ ${ }^{2}$ Institute of Food Technology of Plant Origin, Poznan University of Life Sciences, Poznań, Poland
}

\section{Abstract}

Metabolic syndrome (MetS) is a cluster of metabolic abnormalities that includes dyslipidemia, abdominal obesity, hypertension, and insulin resistance. MetS is more prevalent in postmenopausal women than in other population groups. Furthermore, postmenopausal women are also more prone to nonalcoholic fatty liver disease (NAFLD). The aim of this study was to determine the relationship between fatty acid (FA) status, dietary lipid indices, and noninvasive biomarkers of NAFLD in postmenopausal women with or without MetS.

One hundred and thirty-one postmenopausal women were included in the study. Dietary lipid indices (PUFA/MUFA and n-3/n-6 ratios) were calculated using the mean macronutrient intake, which was evaluated with dietary records. Selected biochemical biomarkers in the blood (e.g., lipid profile and enzymes related to liver function) were measured using the colorimetric method. FA concentrations in red blood cells (RBC) were analyzed using gas chromatography. Noninvasive biomarkers of NAFLD, such as fatty liver index (FLI) and hepatic steatosis index (HSI), were calculated.

The mean age of the study group was $61.48 \pm 5.62$, and the mean waist circumference was $96.27 \pm 12.44 \mathrm{~cm}$. Levels of n-3/n-6 intake exceeding the median were associated with higher levels of EPA, EPA/AA, DHA/AA, and EPA + DHA/AA and lower levels of n-6/ $n-3$ in RBC $(p<0.05)$. Women with MetS had significantly higher $n-6 / n-3$ levels in RBC and lower levels of EPA, total n-3, EPA/ ALA, EPA/AA, DHA/AA, EPA + DHA/AA, PUFA/SFA, and PUFA/MUFA in RBC than women without MetS. Women with MetS had a higher FLI than women without MetS $(p<0.001)$, but there were no differences in the concentrations of enzymes related to liver function. Women with FLI and HSI values over the median and AST/ALT values below the median had lower concentrations of n-3, PUFA/SFA, and PUFA/MUFA in RBC and higher concentrations of n-6/n-3 in RBC ( $\mathrm{p}<0.05)$. Subjects with higher levels of n-3/n-6 and PUFA + MUFA/SFA in RBC had a lower concentration of GGTP, while those with higher levels of PUFA/SFA in RBC had lower FLI $(\mathrm{p}<0.05)$.

MetS is associated with unfavorable FA status in postmenopausal women - specifically with lower levels of n-3, PUFA/SFA, and PUFA/MUFA. Moreover, concentrations of these FAs in RBC are associated with NAFLD biomarkers.

The project was financed by the National Science Centre award (decision number 2015/17/N/NZ9/04133).

\section{Conflict of Interest}

There is no conflict of interest. 